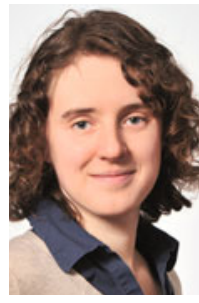

Anna Staroń

Jahrgang 1983. 2002-2007 Biologiestudium an der Universität Warschau, Polen. 2008-2012 Promotion an der LMU München bei Prof. Dr. T. Mascher. Seit 2013 Postdoc am IST Austria in Klosterneuburg, Österreich, in der Arbeitsgruppe von Dr. C. Guet.

DOI: $10.1007 / \mathrm{s} 12268-013-0317-\mathrm{x}$

(C) Springer-Verlag 2013

Bakterien sind ständig wechselnden Umweltbedingungen ausgesetzt: Die physikalisch-chemischen Parameter ändern sich schnell, und Nährstoffmangel ist eher die Regel als die Ausnahme. Um in den sich verändernden Bedingungen zu bestehen, müssen Mikroorganismen die Veränderungen detektieren und sich an sie anpassen. Die extracytoplasmic function(ECF)-Sigmafaktoren zählen neben Ein- und Zweikomponentensystemen zu den Signaltransduktionsmechanismen, die es Bakterien ermöglichen, Änderungen wahrzunehmen und auf sie zu reagieren.

Sigmafaktoren sind Untereinheiten der RNA-Polymerase, die bestimmte DNA-Sequenzen im Promotorbereich erkennen und daran binden. Jeder Sigmafaktor erkennt eine andere DNA-Sequenz und bestimmt dadurch die Promotorspezifität. Die „Haushalts“- oder vegetativen Sigmafaktoren sind essenziell für die Expression der Gene, die für die Aufrechterhaltung des vegetativen Metabolismus wichtig sind. Die alternativen Sigmafaktoren hingegen erkennen Promotoren, die nur unter bestimmten Bedingungen aktiviert werden. $\mathrm{Zu}$ dieser Gruppe zählen auch die ECF-Sigmafaktoren.

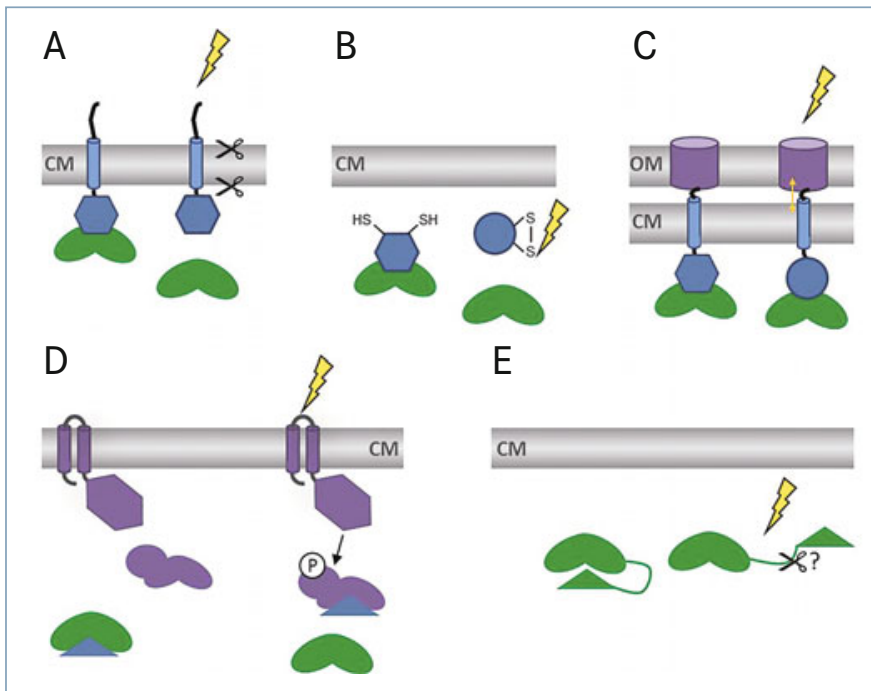

\title{
VAAM-Promotionspreis 2013
}

\section{Vielfalt unter ECF-Sigmafaktoren}

\author{
ANNA STAROŃ \\ INSTITUTE OF SCIENCE AND TECHNOLOGY (IST) AUSTRIA, KLOSTERNEUBURG, \\ ÖSTERREICH
}

Die ECF-Sigmafaktoren werden gewöhnlich unter bestimmten Stressbedingungen aktiviert. In Abwesenheit von Stimuli wird der Sigmafaktor meistens vom Antisigmafaktor gebunden und dadurch inaktiv gehalten. In Anwesenheit eines Stimulus wird der Antisigmafaktor inaktiviert. Die Bakterien verwenden verschiedene Mechanismen, um den Sigmafaktor freizusetzen.

Die alternativen Sigmafaktoren kontrollieren viele wichtige zelluläre Prozesse, beispielsweise die Eisenaufnahme bei Escherichia coli oder Virulenz bei Mycobacterium tuberculosis. Trotzdem sind sie - abgesehen von den wenigen gut beschriebenen Sigmafaktoren in den Modellorganismen - bisher kaum verstanden. Wir haben mittels vergleichender Genomanalyse untersucht und gezeigt, welche Mechanismen der ECF-Sigmafaktor-abhängigen Signaltransduktion unterliegen. Eine ECF-Klassifizierung, die basierend auf der Genomanalyse entwickelt wurde, erlaubt auch eine Vorhersage neuer ECF-abhängiger Mechanismen. Diese Analyse zeigt, dass die ECF-Sigmafaktoren auf sehr unterschiedliche und oft komplexe Weisen den Stimulus wahrnehmen und aktiviert werden können.

Im klassischen Modell zur Aktivierung des ECF-Sigmafaktors wird der Antisigmafaktor nach der Wahrnehmung des Stimulus durch regulierte Proteolyse abgebaut (Abb. 1A). Dieser Mechanismus ist einer der am besten untersuchten und auch relativ weit verbreitet, aber bei Weitem nicht der einzige. Der Antisigma- faktor kann auch durch eine Konformationsänderung inaktiviert werden (Abb. 1B). Manchmal spielen noch zusätzliche Proteine, wie Anti-Antisigmafaktoren, Transmembranproteine oder Zweikomponentensysteme in der Aktivierung der ECFs eine wichtige Rolle (Abb. 1C, D). Interessanterweise findet man auch ECF-Sigmafaktoren, zu denen kein zugehöriger Antisigmafaktor bekannt ist. Diese Gruppe der ECF-Sigmafaktoren ist im Vergleich zu den anderen ECFs deutlich länger. Unsere Studien lassen vermuten, dass diese Verlängerung als eine fusionierte Antisigmaähnliche Domäne fungieren könnte (Abb. 1E).

Sigmafaktoren scheinen eine große Vielfalt an Aktivierungsmechanismen zu benutzen, und wir fangen erst an, sie zu verstehen.

\section{Literatur}

[1] Staroń A, Sofia HJ, Dietrich S et al. (2009) The third pillar of bacterial signal transduction: classification of the extracytoplasmic function (ECF) sigma factor protein family. Mol Microbiol 74:557-581

[2] Staroń A, Mascher T (2010) Extracytoplasmic function factors come of age. Microbe 5:164-170

[3] Wecke T, Halang P, Staroń A et al. (2012)

Extracytoplasmic function - factors of the widely distributed group ECF41 contain a fused regulatory domain.

Microbiologyopen 1:194-213

\section{Korrespondenzadresse:}

Dr. Anna Staroń

Institute of Science and Technology (IST) Austria

Am Campus 1

A-3400 Klosterneuburg

Tel.: +43-(0)2243-9000-4008

anna.staron@ist.ac.at

Sponsoren der drei VAAM-Promotionspreise 2013 waren die Firmen BASF SE, Bayer Pharma AG, Evonik Industries AG, New England BioLabs $\mathrm{GmbH}$ und Sanofi-Aventis Deutschland.
4 Abb. 1: Regulationsschema der Aktivierung von ECF-Sigmafaktoren. Nach der Stimuluswahrnehmung wird der Antisigmafaktor inaktiviert und der Sigmafaktor freigesetzt. Die Inaktivierung des Antisigmafaktors kann mittels (A) Proteolyse, (B) Konformationsänderung, (C, D) Protein-ProteinInteraktion oder (E) Interaktion mit fusionierter Antisigma-ähnlicher Domäne erfolgen. Die Sigmafaktoren sind in Grün, Antisigmafaktoren in Blau, andere Regulatoren in Violett dargestellt. OM, äußere Membran; CM, Zytoplasmamembran (weitere Details siehe Text). 